\title{
Supporting Information: Cluster-weighting in Bulk Phase Vibrational Circular Dichroism
}

\author{
Jan Blasius and Barbara Kirchner* \\ Mulliken Center for Theoretical Chemistry, Rheinische Friedrich-Wilhelms-Universität \\ Bonn, Beringstr. 4+6, D-53115 Bonn, Germany \\ E-mail: kirchner@thch.uni-bonn.de
}




\section{QCE calculations with PEACEMAKER}

Solving the QCE equations is based on an iterative process. This procedure is divided into two iterations of which one is based on the gas phase and the other one on the bulk phase. For the gas phase calculation, a first volume guess is calculated by the ideal gas law and by assuming negligible inter-cluster interactions in the gas phase, the $a_{\mathrm{mf}}$ parameter is set to zero and the cluster partition functions and a first set of cluster populations can be calculated. Afterwards, a new phase volume is obtained by the volume polynomial and the relative volume change is used as a threshold. If the volume change falls below this threshold, the relative Gibbs energy change is calculated and if it falls below a threshold the iteration is assumed to be converged. If the relative volume change exceeds the threshold, the damped initial volume is used for the calculation of the relative Gibbs energy change.

For the liquid phase iteration, the procedure is the same but the starting volume is chosen as the initial gas phase volume divided by 100 to account for the much smaller volume of the bulk. Additionally, the $a_{\mathrm{mf}}$ parameter is not set to zero, as the inter-cluster interactions can not be neglected in the liquid phase. For the case that the gas and liquid phase iteration

converge, the one with the lower Gibbs energy is chosen. For all pairs of $a_{\mathrm{mf}}$ and $b_{\mathrm{xv}}$, the one that yields the smallest deviation from the experimental input is chosen as final solution for the Peacemaker calculation. 


\section{Relative energies}
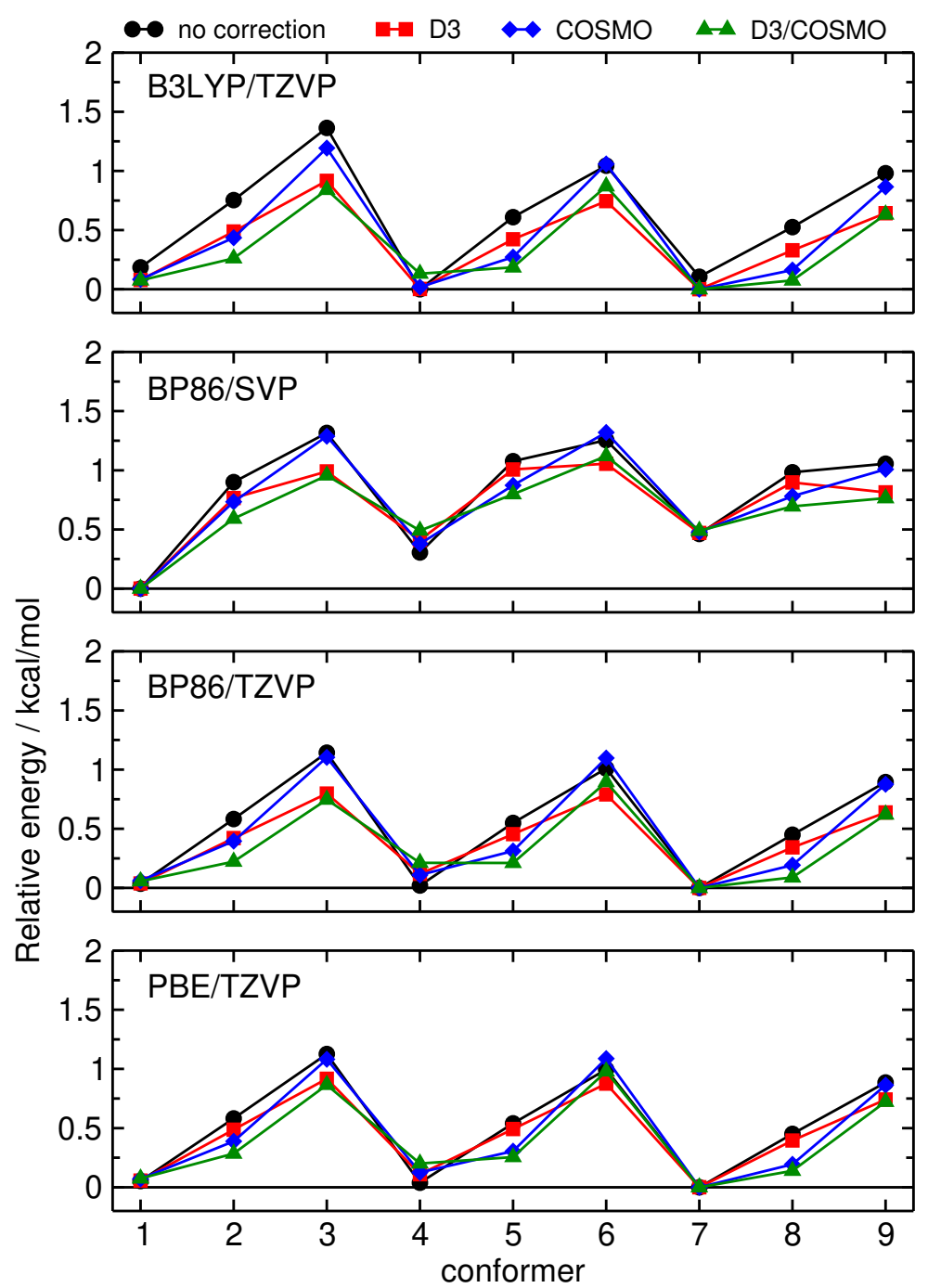

Figure S1: Dependence of the predicted relative energies of the nine conformers on the computational parameters used in the calculations. 

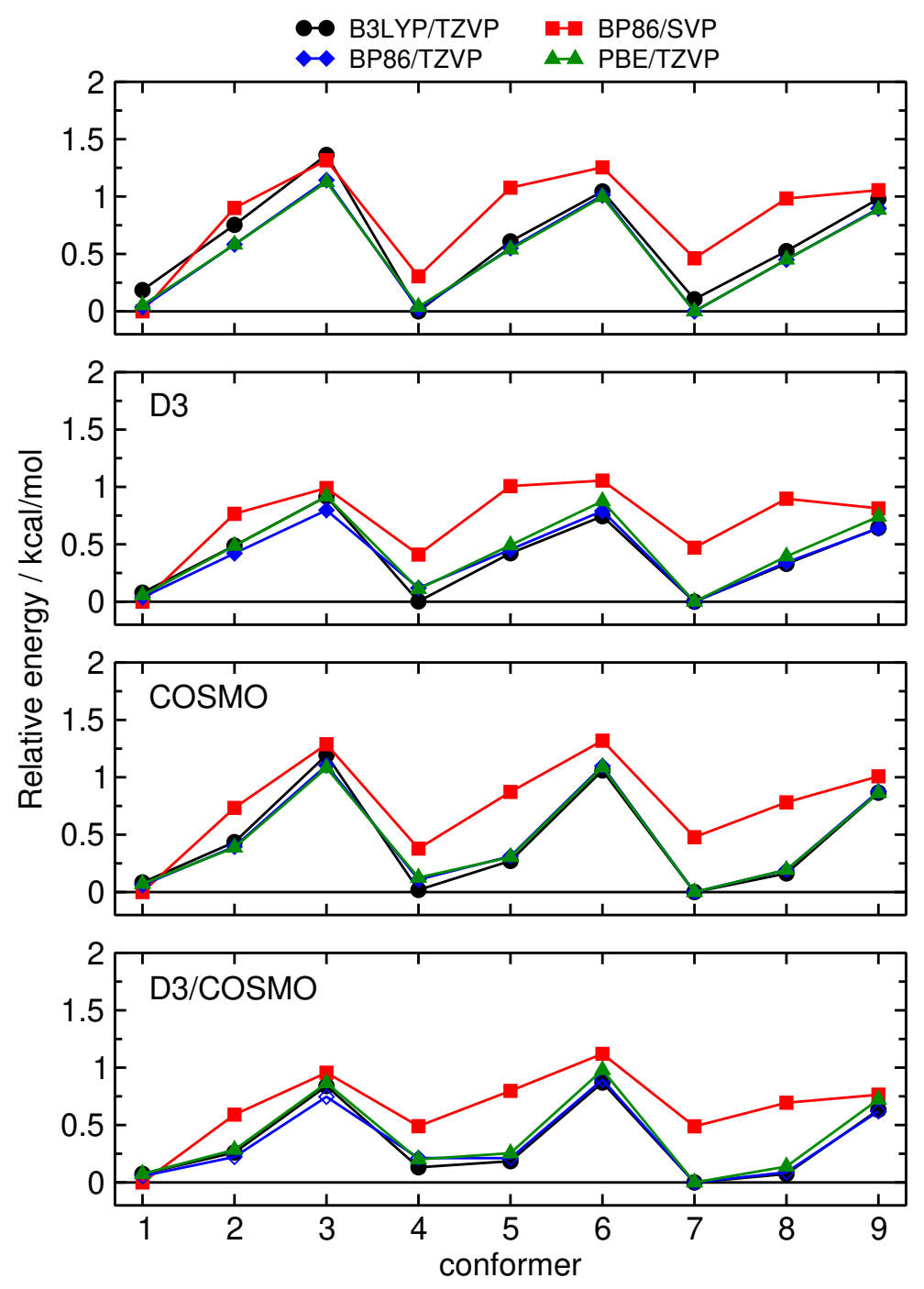

Figure S2: Dependence of the predicted relative energies of the nine conformers on the computational parameters used in the calculations. 


\section{Method test for BW and CW}
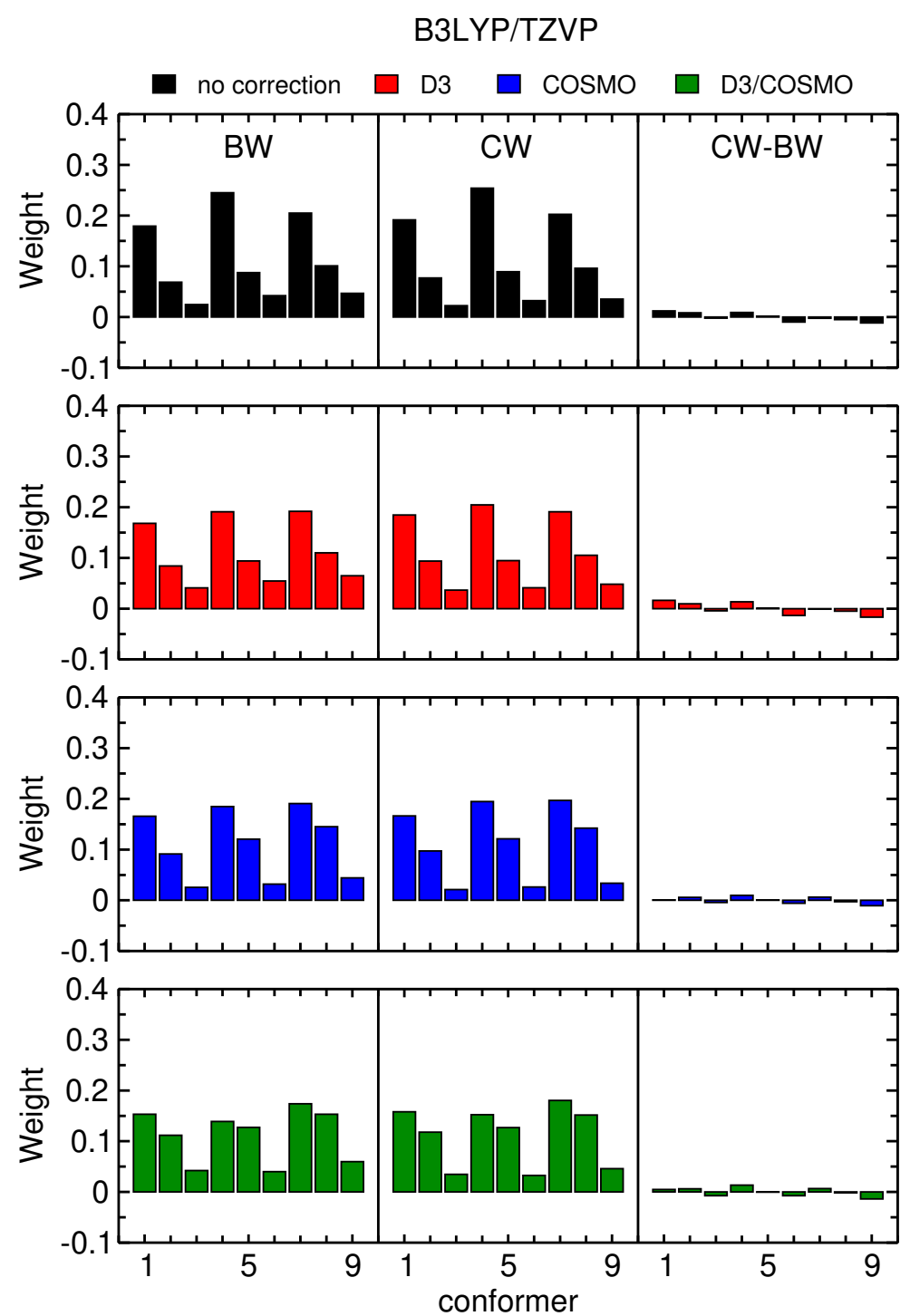

Figure S3: Boltzmann weights (BW) and Cluster weights (CW) as well as different between CW and BW for all $(R)$-Butanol conformers at B3LYP/def2-TZVP level of theory with and without D3 and COSMO. 

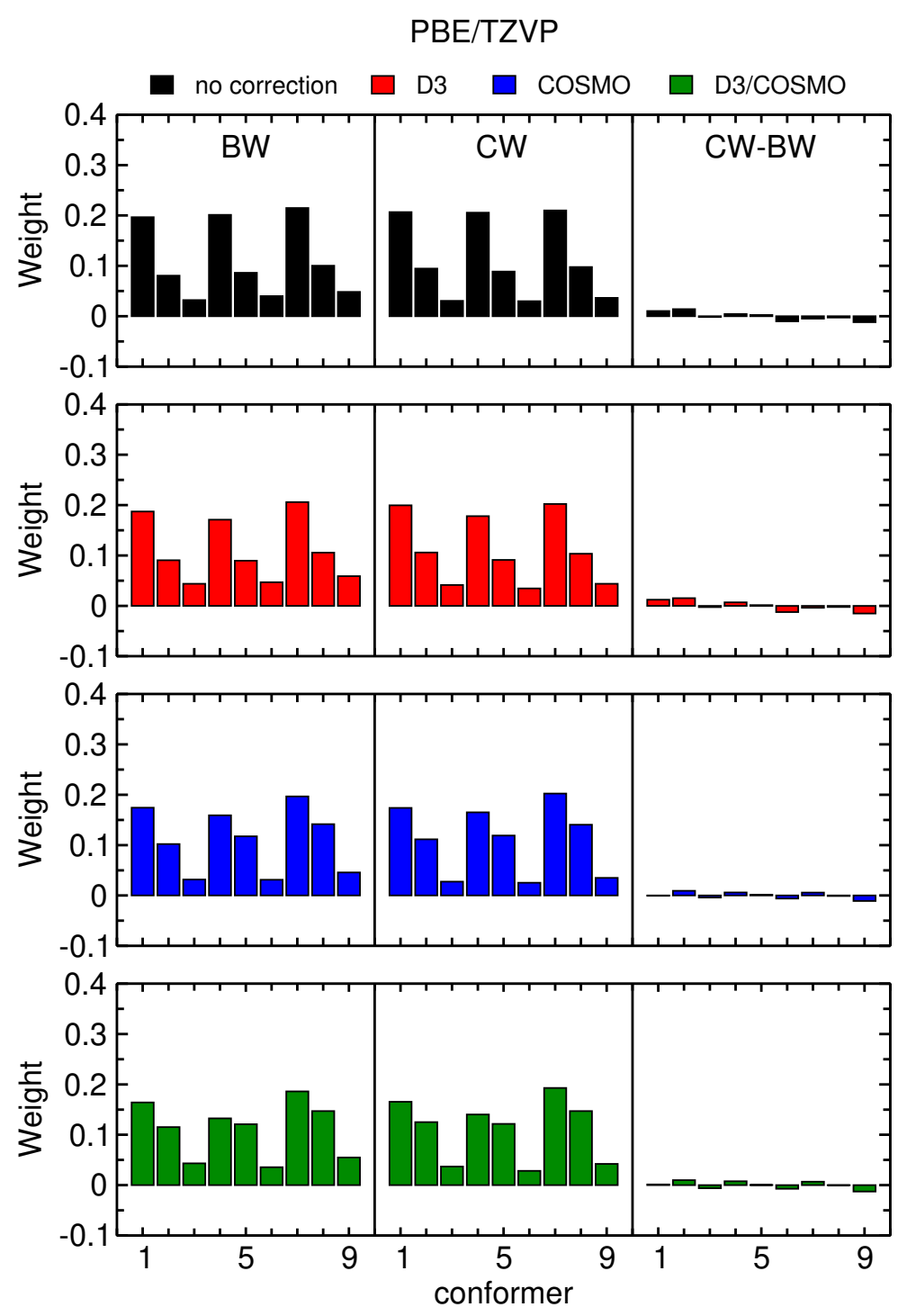

Figure S4: Boltzmann weights (BW) and Cluster weights (CW) as well as different between CW and BW for all $(R)$-Butanol conformers at PBE/def2-TZVP level of theory with and without D3 and COSMO. 


\section{Binding energies of global minima}

Table S1: Binding energies of the global minima calculated at BP86-D3/def2SVP level of theory employing Cosmo. The reference monomer is i1 and the energies are given in units of $\mathrm{kJ} \mathrm{mol}^{-1}$.

\begin{tabular}{c|c|c} 
cluster & $\mathrm{E}_{\text {bind }} / \mathrm{kJ} \mathrm{mol}^{-1}$ & $\mathrm{E}_{\text {bind }}$ per mon. $/ \mathrm{kJ} \mathrm{mol}^{-1}$ \\
\hline ii1 & -39.971 & -19.985 \\
iiia1 & -102.194 & -34.065 \\
iva1 & -176.209 & -44.052 \\
va1 & -213.242 & -42.648 \\
via1 & -268.158 & -44.693 \\
viia1 & -329.169 & -47.024 \\
viiia1 & -384.284 & -48.036 \\
ixa1 & -443.870 & -49.319 \\
xa1 & -509.393 & -50.939
\end{tabular}




\section{Weighted monomer spectra employing Cosmo}

Table S2: Overlap between the experimental gas phase and theoretically predicted VCD spectra based on BW and CW using monomer conformers. "Solv" indicates the usage of Cosmo. The set I-1 includes i1 (def2-SVP) or i7 (def2TZVP), I-5 includes i1, i2, i4, i7 and i8, I-9 includes all conformers.

\begin{tabular}{c|c|cccccc}
\hline Weight & Solv & \multicolumn{3}{|c}{ def2-SVP } & \multicolumn{3}{c}{ def2-TZVP } \\
\hline & & I-1 & I-5 & I-9 & I-1 & I-5 & I-9 \\
\hline BW & no & 0.264 & 0.413 & 0.398 & 0.313 & 0.435 & 0.435 \\
BW & yes & 0.181 & 0.239 & 0.208 & 0.245 & 0.195 & 0.159 \\
CW & no & 0.264 & 0.431 & 0.397 & 0.313 & 0.432 & 0.424 \\
CW & yes & 0.181 & 0.249 & 0.204 & 0.245 & 0.197 & 0.159 \\
\hline
\end{tabular}




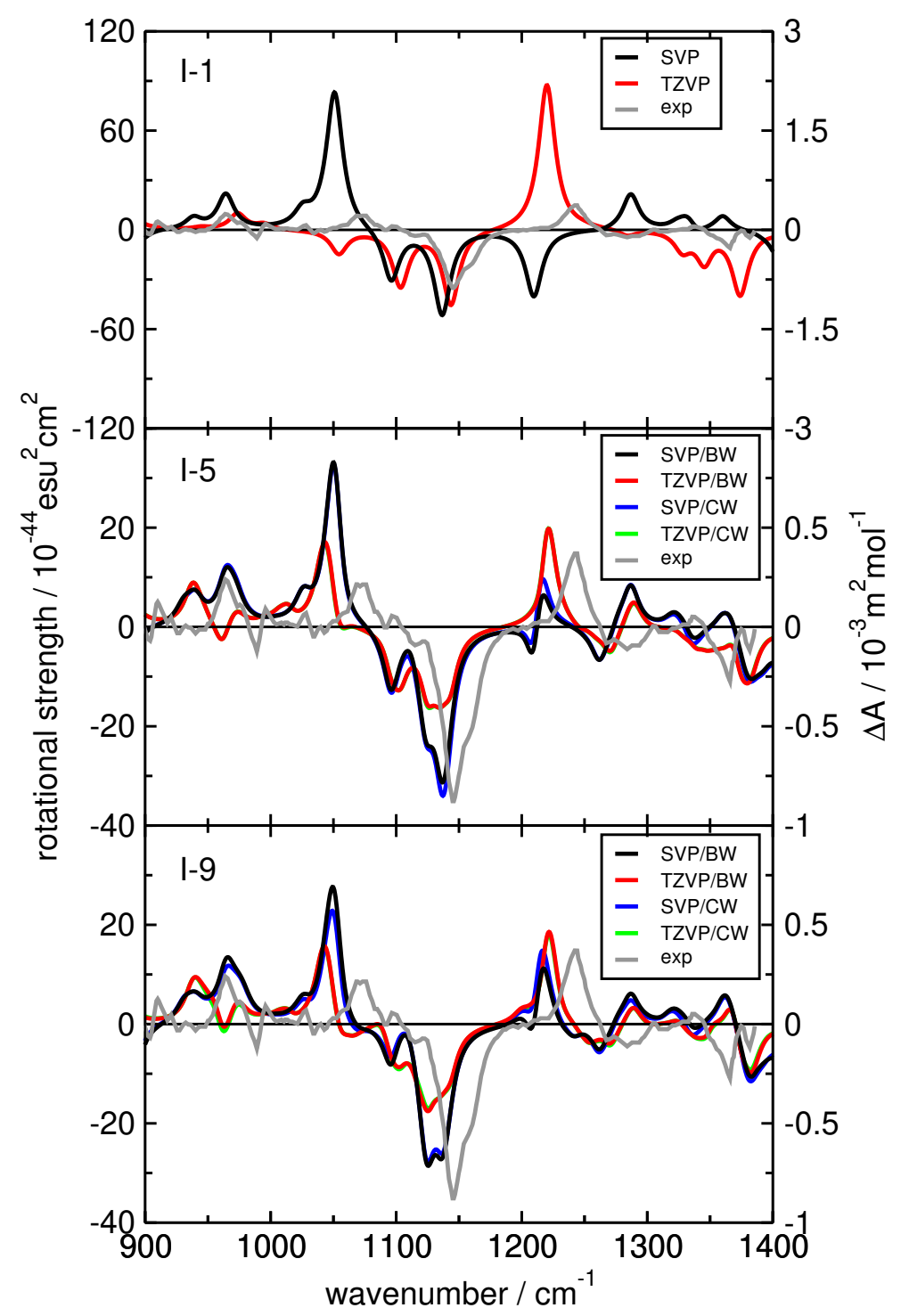

Figure S5: Calculated VCD spectra based on the cluster sets (I-1), (I-5) and (I-9) obtained by weighting with BW as well as CW for the def2-SVP and def2-TZVP basis set with employing implicit solvation. The grey lines depict the experimental reference measured for a $0.029 \mathrm{M}$ $(R)$-2-butanol solution in carbon disulfide. ${ }^{1}$ 


\section{Exlcuding single global minima}

Table S3: Single clusters are excluded from the S4${ }^{\mathrm{gm}}$ set and the overlap between experimental bulk and CW weighted VCD spectra is calculated.

\begin{tabular}{cc|ccccccccc} 
& & \multicolumn{1}{|c}{ excluded cluster } \\
Solv & Phase & ii1 & iiia1 & iva1 & va1 & via1 & viia1 & viiia1 & ixa1 & xa1 \\
\hline yes & bulk & 0.045 & 0.045 & 0.326 & 0.045 & 0.045 & 0.045 & 0.045 & 0.045 & 0.045
\end{tabular}




\section{Optimized QCE parameter for all cluster sets employed}

Table S4: Optimized QCE parameters $a_{\mathrm{mf}}$ and $b_{\mathrm{xv}}$ for the clustersets employing structures optimized without employing Cosmo. The level of theory is BP86D3/def2-SVP or BP86-D3/def2-TZVP. The parameter $a_{\mathrm{mf}}$ is given in units of $\mathrm{J} \mathrm{m}^{3} \mathrm{~mol}^{-2}, b_{\mathrm{xv}}$ has no unit.

\begin{tabular}{c|cc} 
set & $a_{\mathrm{mf}}$ & $b_{\mathrm{xv}}$ \\
\hline$(\mathrm{I}-5)(\mathrm{SVP})$ & 1.976 & 0.799 \\
$(\mathrm{I}-9)(\mathrm{SVP})$ & 1.976 & 0.799 \\
$(\mathrm{I}-5)(\mathrm{TZVP})$ & 1.976 & 0.799 \\
$(\mathrm{I}-9)(\mathrm{TZVP})$ & 1.976 & 0.799
\end{tabular}

Table S5: Optimized QCE parameters $a_{\mathrm{mf}}$ and $b_{\mathrm{xv}}$ for the clustersets employing structures optimized with employing Cosmo. The level of theory is BP86D3/def2-SVP with Cosmo employed as implicit solvation model. The parameter $a_{\mathrm{mf}}$ is given in units of $\mathbf{J ~ m}^{3} \mathbf{m o l}^{-2}, b_{\mathrm{xv}}$ has no unit.

\begin{tabular}{c|cc|c|cc|c|cc|c|cc}
\multicolumn{4}{c}{ glob. min. } & \multicolumn{2}{c}{ glob. isom. min } & \multicolumn{1}{c}{ all } \\
\hline set & $a_{\mathrm{mf}}$ & $b_{\mathrm{xv}}$ & set & $a_{\mathrm{mf}}$ & $b_{\mathrm{xv}}$ & set & $a_{\mathrm{mf}}$ & $b_{\mathrm{xv}}$ & set & $a_{\mathrm{mf}}$ & $b_{\mathrm{xv}}$ \\
\hline $\mathrm{S} 1^{\mathrm{gm}}$ & - & - & - & - & - & $\mathrm{S}^{0.01}$ & 1.976 & 0.799 & $\mathrm{~S} 1^{\text {all }}$ & 1.976 & 0.799 \\
$\mathrm{~S}^{\mathrm{gm}}$ & 1.599 & 0.836 & - & - & & $\mathrm{S}^{0.01}$ & 1.611 & 0.837 & $\mathrm{~S} 2^{\text {all }}$ & 1.661 & 0.838 \\
$\mathrm{~S}^{\mathrm{gm}}$ & 1.355 & 0.851 & $\mathrm{~S}^{\text {gim }}$ & 1.337 & 0.851 & $\mathrm{~S} 3^{0.01}$ & 1.315 & 0.850 & $\mathrm{~S} 3^{\text {all }}$ & 1.349 & 0.851 \\
$\mathrm{~S}^{\mathrm{gm}}$ & 0.772 & 0.836 & $\mathrm{~S}^{\text {gim }}$ & 0.772 & 0.836 & - & - & - & $\mathrm{S} 4^{\text {all }}$ & 1.236 & 0.860 \\
$\mathrm{~S}^{\mathrm{gm}}$ & 1.374 & 0.883 & $\mathrm{~S}^{\text {gim }}$ & 1.374 & 0.883 & - & - & - & $\mathrm{S} 5^{\text {all }}$ & 1.350 & 0.851
\end{tabular}


Cluster spectra for set $\mathrm{S}^{\text {gim }}$
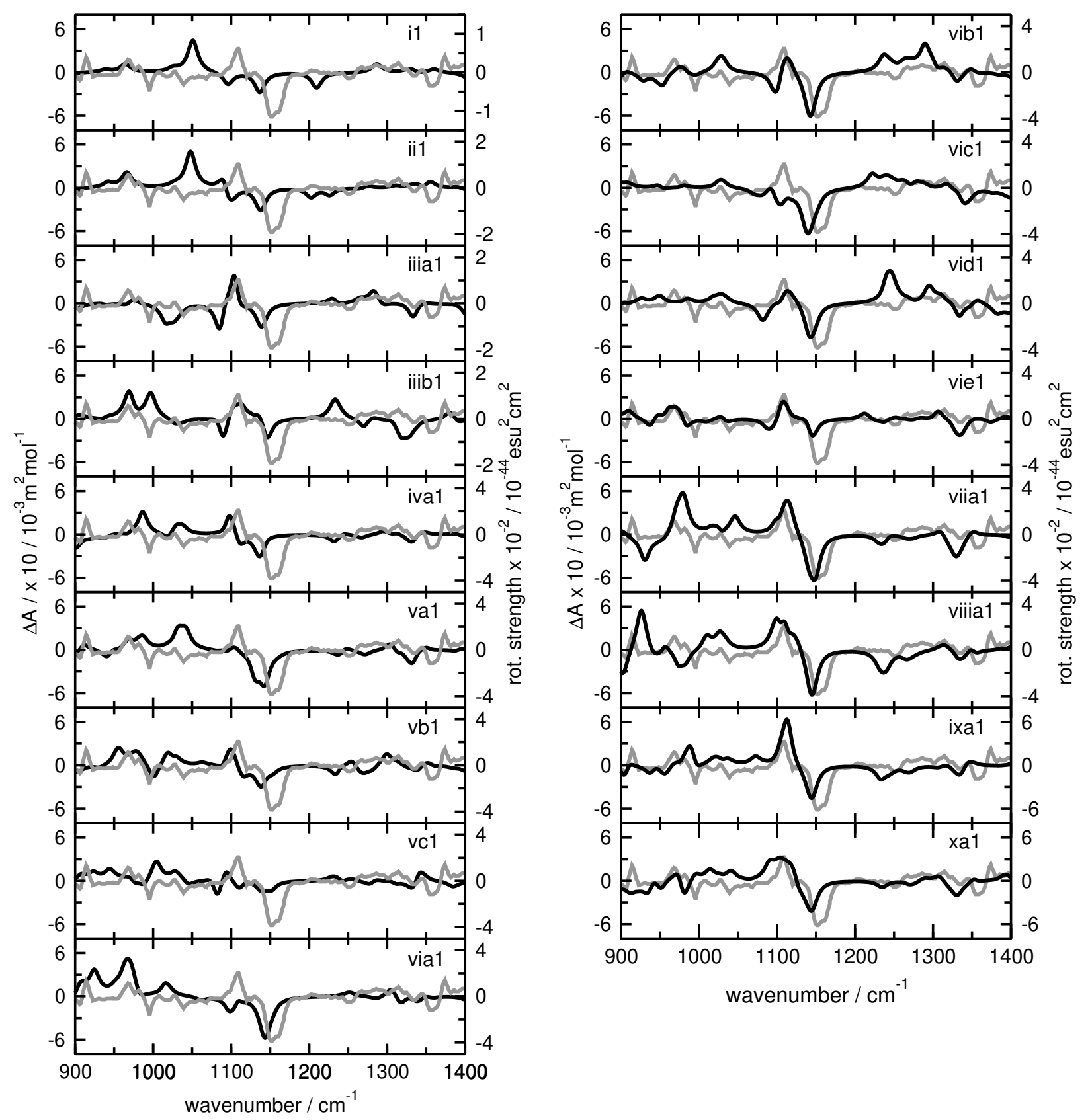

Figure S6: Comparison between the individual VCD spectra of the clusters included in the $\mathrm{S} 4^{\text {gim }}$ cluster set and the experimental reference for the bulk phase. Calculated spectra are shown in red, black depicts the experimental reference. ${ }^{1}$ 


\section{Cluster set labels and clusters included in the sets}

Table S6: Cluster set labels and included number of clusters in the respective sets.

\begin{tabular}{|c|c|}
\hline set label & clusters included \\
\hline $\mathrm{S} 1^{\mathrm{gm}}$ & $(\mathrm{I}-1)$ \\
\hline $\mathrm{S} 1^{0.01}$ & $(\mathrm{I}-5)$ \\
\hline $\mathrm{S} 1^{\text {all }}$ & $(\mathrm{I}-9)$ \\
\hline $\mathrm{S} 2^{\mathrm{gm}}$ & $(\mathrm{I}-1 ; \mathrm{II}-1)$ \\
\hline $\mathrm{S} 2^{0.01}$ & (I-5;II-23) \\
\hline $\mathrm{S} 2^{\text {all }}$ & $(\mathrm{I}-9 ; \mathrm{II}-69)$ \\
\hline $\mathrm{S} 3^{\mathrm{gm}}$ & (I-1;II-1;IIIa-1) \\
\hline S3 $3^{\text {gim }}$ & (I-1;II-1;IIIa-1;IIIb-1) \\
\hline $\mathrm{S} 3^{0.01}$ & (I-5;II-23;IIIa-21) \\
\hline S3 $3^{\text {all }}$ & (I-9;II-69;IIIa-121;IIIb-118) \\
\hline $\mathrm{S} 4^{\mathrm{gm}}$ & (I-1;II-1;IIIa-1;IVa-1;Va-1;VIa-1;VIIa-1;VIIIa-1;IXa-1;Xa-1) \\
\hline $\mathrm{S} 4^{\text {gim }}$ & (I-1;II-1;IIIa-1;IIIb-1;IVa-1;Va-3;VIa-7;VIIa-1;VIIIa-1;IXa-1;Xa-1) \\
\hline $\mathrm{S} 4^{\text {all }}$ & (I-9;II-69;IIIa-121;IIIb-118;IVa-1;Va-3;VIa-7;VIIa-1;VIIIa-1;IXa-1;Xa-1) \\
\hline $\mathrm{S} 5^{\mathrm{gm}}$ & (I-1;II-1;IIIa-1;Va-1;VIa-1;VIIa-1;VIIIa-1;IXa-1;Xa-1) \\
\hline $\mathrm{S} 5^{\mathrm{gim}}$ & (I-1;II-1;IIIa-1;IIIb-1;Va-3;VIa-7;VIIa-1;VIIIa-1;IXa-1;Xa-1) \\
\hline S5 $5^{\text {all }}$ & (I-9;II-69;IIIa-121;IIIb-118;Va-3;VIa-7;VIIa-1;VIIIa-1;IXa-1;Xa-1) \\
\hline
\end{tabular}

\section{Acknowledgment}

The authors thank the DFG under the grant project number 406232243.

\section{References}

(1) Wang, F.; Polavarapu, P. L. Vibrational circular dichroism: Predominant conformations and intermolecular interactions in (R)-(-)-2-butanol. J. Phys. Chem. A 2000, 104, 10683-10687. 